\title{
Téoros
}

Revue de recherche en tourisme

\section{Les aéroports de Montréal : un processus de prise en charge laborieux}

\section{Marcel Le Sieur}

Volume 10, numéro 3, novembre 1991

Destination Montréal : d'hier à demain

URI : https://id.erudit.org/iderudit/1079195ar

DOI : https://doi.org/10.7202/1079195ar

Aller au sommaire du numéro

Éditeur(s)

Université du Québec à Montréal

ISSN

0712-8657 (imprimé)

1923-2705 (numérique)

Découvrir la revue

Citer cet article

Le Sieur, M. (1991). Les aéroports de Montréal : un processus de prise en charge laborieux. Téoros, 10(3), 6-8. https://doi.org/10.7202/1079195ar d'utilisation que vous pouvez consulter en ligne.

https://apropos.erudit.org/fr/usagers/politique-dutilisation/ 


\section{Les aéroports de Montréal: un processus de prise en charge laborieux}
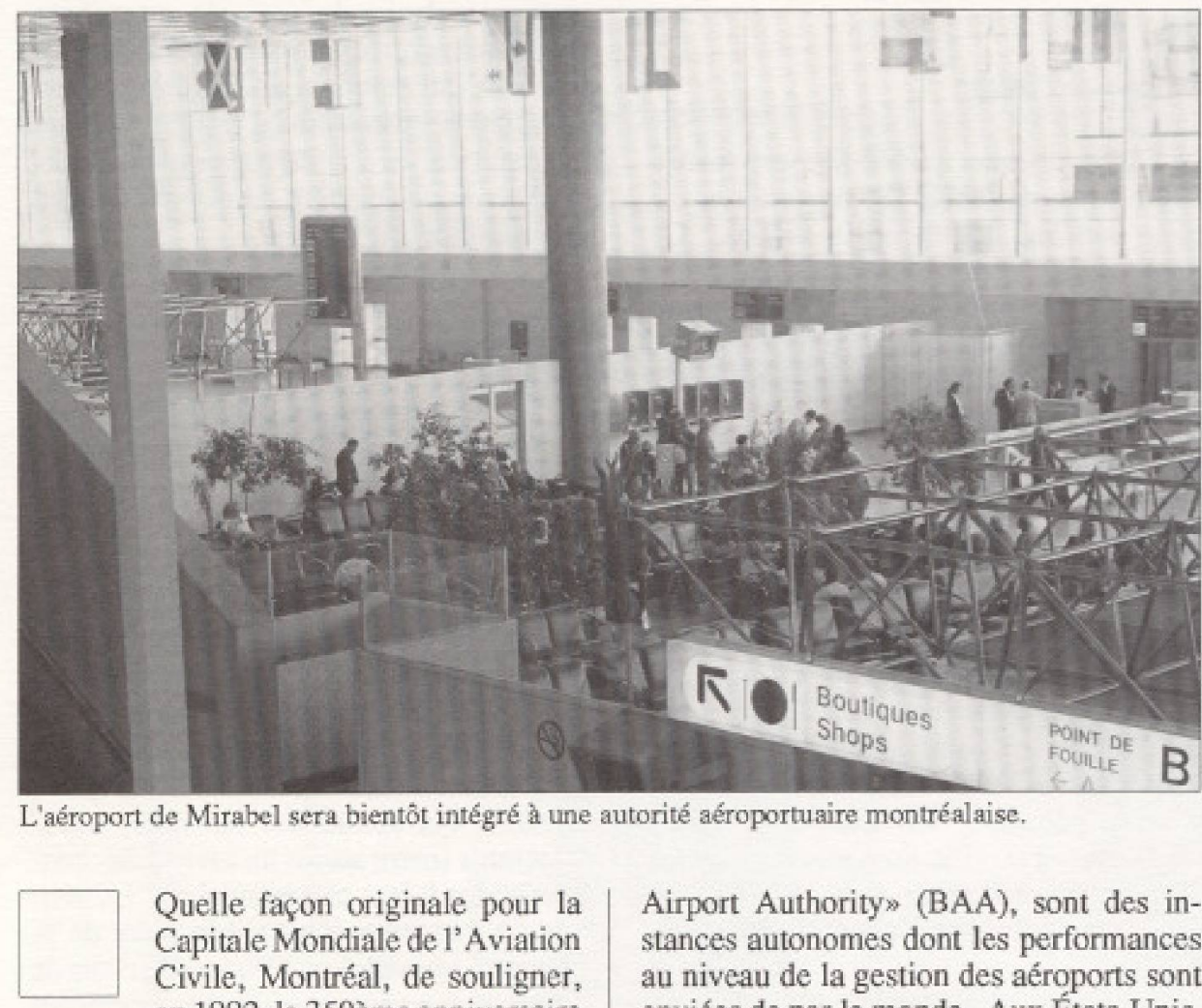

aéroport de Mirabel sera bientōt intégré à une autorité aéroportuaire montréalaise.

Quelle façon originale pour la Capitale Mondiale de $l^{\prime}$ Aviation Civile, Montréal, de souligner, en 1992, le 350ème anniversaire de sa création en marquant un tournant majeur de son histoire grâce à la prise en charge de ses aéroports internationaux par une autorité aéroportuaire locale. Après plus de cinq années de démarches, cette transition s'inscrira comme l'une des premières au Canada, imitée seulement, en première phase, par les villes de Vancouver, Edmonton et Calgary. Toutefois, bien qu'au Canada, Montréal fasse figure de pionnière en ce domaine, comme elle l'a d'ailleurs toujours été dans chacun des modes du transport, on ne peut cependant pas considérer que le geste de créer une autorité aéroportuaire locale pour prendre en charge ses infrastructures constitue un précédent significatif à l'échelle du globe. En effet, cette façon de gérer les aéroports nationaux a été adoptée, il y a belle lurette, par une majorité de pays dans le monde. ADP ou Aéroports de Paris, la «British

\footnotetext{
Monsieur Marcel Le Sieur est Commissaire principal a la CIDEM rvile de Montréal) et representant de la Vila de Montréal au Conseil de la SOPRAM ISociéte de promation des aéroports de Montréall.
}

Airport Authority* (BAA), sont des instances autonomes dont les performances au niveau de la gestion des aéroports sont enviées de par le monde. Aux Êtats-Unis, il y a dejà plus de quinze ans que le gouvernement fédéral, la FAA, a renoncé à la gestion du demier aéroport fédéral et en a transféré l'administration et le contrôle à une autorité locale.

Pourquoi un tel retard par rapportàce qui se fait ailleurs dans le monde? et pourquoi Montrếal tient-elle tant à prendre en charge ses infrastructures acroportuaires? Telles sont les deux questions qui ont été maintes fois soulevees.

\section{Un premier essai, à l'ère de la déréglementation}

En ce qui concerne tout d'abord le retard de Montréal, il fautse rappeler qu'on en entend parlerdepuis une vingtaine d'années. Déjà, après la décision de 1969 de construire un second aéroport international pour la région de Montréal, les études effectuées pour le compte des instances fédérales recommandaient que la gestion *autonome» ou "privếe» de cette nouvelle infrastructure soit sérieusement considérée. D'autres études, effectuées dans les années subséquentes pour des entités provinciales et régionales, en arrivaient à des conclusions similaires. Mais cette idée, qui équivalait au démembrement de l'empire que s'était constitué Transport Canada à travers le pays, a régulièrement ét battue en brèche par lesmandarins du Ministère qui rejetaient la perte anticipée de la gestion de milliers de fonctionnaires et de millions de dollars de budget que représentait l'administration de près de 200 aéroports fédéraux à travers le Canada.

C'est une volonté politique du gouvernement conservateur, inspirée par l'exemple de la déréglementation aux Etats-Unis, qui a produit, en 1979, le rapport Haglund, lequel recommandait au gouvernement canadien de favoriser le transfert de l'administration et du contrôle des principaux aéroports canadiens à des autorités aéroportuaires locales. Mais la brièveté de ce gouvernement et l'opposition traditionnelle des gestionnaires du ministère des Transports ont vite fait reléguer aux oubliettes ce rapport et ses conclusions révolutionnaires.

Les recommandations de ce rapport ne sont cependant pas restées lettre morte. Elles ont retenu l'attention d'individus qui, tant dans la région de Montréal qu'ailleurs au Canada, déploraient la centralisation excessive des pouvoirs de Transports Canada à Ottawa et la concentration croissante de l'activité aćrienne canadienne à l'aéroport Pearson de Toronto. Malgré les réticences du gouvernement libéral à donner suite à des recommandations émanant d'un rapport produit à la demande des conservateurs, et malgré également la toute-puissance des hautes autorités de Transports Canada qui résistaient farouchement à une dilution de leurs pouvoirs, le cabinet fédéral acceplait, en juin 1984, de tenter l'expérience de confier la gestion de l'aéroport de Mirabel à une société de la couronne locale, la Sociéteté Immobilière du Canada (Mirabel) Ltée. Toutefois, on recommandait alors d'attendre une période plus propice, à 
l'autome 1984, pour en faire l'annonce et la mise en oeuvre en grandes pompes! Or, le 4 septembre 1984, le gouvemement conservateur reprenait le pouvoir.

Cette arrivec au pouvoir du gouvemement conservateur a, certes, tué dans l'oeuf le projet d'expérience pilote à Mirabel mais n'a pas pour autant condamné à jamais l'espoir des instances régionales concernées. Des son arrivéte au pouvoir, le ministre des Transports de l'époque, Don Mazankows$\mathrm{ki}$, décidait de ressusciter le rapport Haglund et de le faire traduire en propositions concretes de mise en oeuvre à l'echelle du pays. Pour ce faire, il nomma un comité formé de représentants dechaque région du Canada, charge d'exprimer les attentes locales de rocommander les mécanismes lesplus appropriés de transfertéventuel des aéroports canadiens.

\section{Transports Canada résiste..}

Or, il est tres symptomatique de réa liserque ce groupe de travail a $e_{t}$ à ce point influencé par les deux hauts fonctionnaires de Transports Canada qui le chapeautaient, madane Janet Snith et monsieur Dave McAree, que le premier rapport qu'ils ont produit recommandait que la meilleure solution d'avenir pour la gestion des aéroports canadiens bait le maintien d'une administration aćroportuaire par un ministère des Transports Canada amélioré, et ce, malgré les constats contraires des études commandées et les avis divergents des menbres civis du comité. Le ministre John Crosbie, à qui ce premier rapport fut

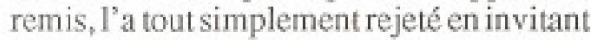
les auteurs a respecter les recommandations des études et les avis rếgionaux exprimés. Curieusement, le rapport final du groupe en arriva à la conclusion que la meilleute solution, pour gếrer les aéroports, était la création d'autorités aéroportuaires locales.

En mêne temps que le ministre Mazankowski relançait les principes du rapport Haglund et jetait los bases de sa politique Aller sans entraves, c'est-â-dire dès l'automne 1984 , son collègue, le ministre d'Etat aux Transponts, Benoît Bouchard, nommait un comité consultatif local, le Conseil Consultatif des Aéroports de Montreal, preside a l'origine par Monsieur Pierre MacDonald alors vice-president de la Chambre de Commerce de Montréal. afin de proposer des moyens $\mathrm{d}^{+}$améliorer le marketing et le développement des aéroports montréalais. Or, à peine ce Conseil avait-il débuté ses travaux, que le président d'Air Canada, Pierre Jeanniot, déclarait, à la une des journaux: *Il faut fermer Mirabels. Cette déclaration fracassante provoqua la modification de la vocation du Conseil à qui on confia alors le mandat de se pencher sur $1^{+}$avenir des deux aeroports montréalais et de faire des recommandations approprices.

C'est ainsi que le groupe du Conseil Consultatif entreprit de nombreuses études et analyses, plus d'une quarantaine, résumées dans un rapport, connu sous le vocable de Rapport Sauriol, remis au ministre fedstal des Transports en septembre 1986. La principale conclusion de ce rapport te. moignait d'une division marquée de la communauté montréalaise à l'eggard du choix d'un seul aéroport pour la région. En effet, malgré la démonstration des effets néfastes du maintien de deux aéroports, on n'arrivait pasà un consensus sur le choix de Mirabel ou Dorval.

On se souvient que cet affrontement entre les partisans de la concentration dans le nouvel aéroport de Mirabel et les partisans du retour à l'aéroport unique de Dorval survenait alors pour la troisieme reprise en dix ans. Il avait eu lieu la première fois en 1975 lors de l'ouverture de Mirabel qui devait devenir alors le principal aéroport de Montréal. A cette époque, les gens qui avaient réclamé, en 1967 , la fermeture de Dorvalà cause du bruit, et forcé la construction d'un nouvel aéroport à Mirabel, ne voulaient plus du tout voir disparaitre leur aćroport. Cette tergiversation a retardé la finalisation des travaux qui devaient assurer à Mirabel sa pleine autonomic (autoroute 13, transport rapide par train, transfert de tous les vols, etc.). Cinq ans plus tard, en 1981, à la date prévue pour une seconde phase de transfert de vols de Dorval vers Mirabel, les partisans des deux aéroports s'affrontèrentencore une fois sans. résultats. Tout comme lors de la troisione remise en question en 1986, les politiciens refuserent de trancher le debat et de prondre une decision lourde de consequences. Pendant ce temps toutefois, Toronto profitait outrageusement du climat d'incertitude qui caractérisait le dossier montréalais et on a assisté à un déplacenent considérable du trafic aérien jusqu'alors concentró à Montréal. Pourtant, le 4 décembre 1986, le gouvernement fédếral refusait encore une fois de trancher entre Dorval et Mirabel et annonçait le maintien d' un aéroport à deux terminawx..!' (sic) pour Montréal.

\section{Un consensus montréalais se manifeste}

Mais en rendant publique cette indécision, le gouvernement fédéral oubliait du mềme coup la seconde recommandation du rapport Sauriol, celle portant sur la structure de gestion proposé pour les aeroports de Montrâal. Cette recommandation proposant le transfert des aéroports à une corporation locale avait été pourtant unanime. $11 \mathrm{n}^{*} \mathrm{en}$ fallait pas plus pour que les montréalais refassent rapidement consensus autour de la réclamation d'instauration d'une autorité aéroportuaire locale. C'est la Ville de Mon tréal qui pril le leadership de ce dossier dés le début de l'année 1987. Avec ses partenaires de la CUM, de la Chambreet du Bureau de Commerce qui avaient tous vecu et constaté les conséquences désastreuses des trois affrontements antérieurs sur le choix de Dorval ou de Mirabel, le maire de Montréal proposa que cette question de la fermeture de l'un ou l'autre des deux aéroports ne soit plus jamais remise sur la table et que tous devaient s'unir pour réclamer le transfert de la gestion des deux aéroports à une instance locale qui les développerait à l'abri du dirigisme d'Otawa.

La rapidité avec laquelle les différentes instances de la région de Montréal ont refait un consensus autour de cette question a complètement pris de court les hauts fonctionnaires de Transports Canada et l'entourage du ministre John Crosbie qui avait accepté de recevoir une délégation de la région de Montréal le 5 mars 1987.

C'est donc avec le maire Jean Doré à sa têté que cette délégation de présidents et viceprésidents de la Chambre de Commerce, du Bureau de Commerce, de la CUM et de la Conférence des Maires de la Banlieue a rencontré le ministre Crosbie ce 5 mars 1987 pour lui annoncer la volonté unanime de tous les intervenants montréalais à ré́clamer la cré̉ation immédiate d'une autorité aéroportuaire locale et le transfert des deux aéroports à cette dernière. C'était la première fois que le gouvernement fédéral faisait face à un groupe représentatif aussi déterminé et aussi bien documenté sur les orientations politiques gouvernementales issues des nombreux rapports antérieurs concernant la gestion des aéroports. 
Surpris par la détermination du maire de la plus grande ville canadienne et du support unanime de sa communauté, le ministre Crosbie publiait, à peine un mois plus tard, soit le 9 avril 1987 , une politique intitulée «Le futur cadre de gestion des aéroports canadiens* dans laquelle le gouvernement du Canada fixait les grandes lignes d'un processus visant I'éventuelle création d'autorités aéroportuaires locales et les mécanismes de la prise en charge des aéroports de lẹr communauté. Très rapidement, la communautể montréalaise faisait savoir au Ministre, par la bouche du maire Doré, qu'elle acceptait les grandes lignes de cette politique, qu'elle refusait la mise en place de comités consultatifs transitoires nommés par le fédéral, et qu'elle entreprenait immédiatement la constitution de sa corporation aéroportuaire.

C'est donc ainsi que vit le jour, au printemps 1987, «Le Conseil de l'Aéroport International de Montréals. Fort de l'expérience du précédent conseil consultatif de 1984 dont les membres avaient été nommés par Ottawa, qui ne représentaient pas proportionnellement toute la région et qui étaient, pour certains, en conflit d'intérêt, il fut cente fois décidé que ce nouveau conseil serait à l'abri de toute critique. Ainsi, après que le maire de Montréal se soit assuré des appuis politiques des maires de la rive Nord et de la rive Sud, on s'est entendu sur la désignation des sept partenaires qui représenteraient le mieux, démocratiquement et démographiquement, l'ensemble de la grande région montréalaise. Les sept instances désignées furent, et sont encore, les suivantes:

- la Ville de Montréal;

- la Conférence des Maires de la Banlieue de Montréal;

- laChambre de Commerce du Montréal Métropolitain;

- le Bureau de Commerce de Montréal; la Ville de Laval;

- un groupe de maires de la Rive Nord avec le COPAM(Comité de promotion de l'aéroport à Mirabel);

un groupe de maires de la Rive Sud avec la SMD (Société Montérégienne de Développement).

Chacune de ces instances fut invitée à désigner deux (2) rêprésentants, un élu et un représentant administratif. Le Conseil de quatorze membres ainsi constitué a commencé ses travaux en juin 1987.
Durant l'été de 1987, ces membres se sont penchés sur le raffinement de la stratégie d'intervention, sur la consolidation du consensus régional etsurla réalisation d'une charte pour le Conseil. Mais surtout, ils ont procédé à la sélection de personnes d'affaires afin de constituer un comité exécutif ou si on veut, un conseil d'administration du Conseil. Ces gens d'affaires, choisis parchacune des instances membres tant pour leur expertise que pour leur absence de conflit $\mathrm{d}^{\prime}$ 'intérềt actuel et potentielavec 1'opération aéroportuaire, ont été nommés en même temps qu'entraient en vigueur les Lettres Patentes du Conseil de l'Aéroport International de Montréal, soit le 4 décembre 1987.

\section{Une autorité aéroportuaire montréalaise en place... ou presque!}

Mandatés pour entreprendre le processus de négociation de la prise en charge des aéroports, ils ont obtenu, en premier lieu, leur reconnaissance officielle, par les deux paliers de gouvernement, comme l'unique entité dûment mandatée et reconnue pour représenter l'ensemble de la grande région montréalaise. Ensuite, nantis d'un financement fourni par les partenaires montréalais ainsi que par Ottawa et Québec, ils ont entrepris les études et analyses devant permettre un examen complet du systeme administratif et financier des aéroports de Montréal. Cette opération a permis de déposer, dès le mois d'août 1988, une proposition concrète d'achat ou de location avec option d'achat des aéroports au gouvernement fédéral.

Il a fallu attendre un an la réponse du gouvernement fédéral qui signait enfin, le 31 juillet 1989 , un protocole d'entente acceptant en principe la proposition montréalaise et autorisant ainsi l'amorce du processus beaucoup plus rigoureux $\mathrm{d}^{\dagger}$ analyse et de négociation devant aboutir à un transfert intégral de la gestion des aéroports. Depuis donc plus dedeux longues années, le Conseil de l'Aéroport International de Montréal qui, depuis, s'est scindé en deux corporations, l'uneàcharte fédérale, ADM ou Aéroports de Montréal chargée de la négociation à titre d'Autorité Aéroportuaire locale, et l'autre, la SOPRAM ou Société de Promotion des Aéroports de Montréal qui a repris la charte provinciale duprécédentConseil, a investidescentaines de milliers de dollars dans la préparation et la négociation des volumineux textes d'ententes avec le fédéral.

Malgré la lenteur désespérante qui caractérise l'approche gouvernementale dans ce dossier, et qui illustre de façon particulièrement éloquente la raison fondamentale qui incite les partenaires montréalais à tant vouloir se doter d'une structure de décision locale afin de mieux gérer et développer nos aéroports, deux gestes significatifs ont puêtre posés jusqu'ici. En avril 1991, ADM et Transports Canada se sont entendus sur le contenu d'une première entente financière fixant les paramètres monétaires du bail à venir.

Par lasuite, le 15 mai 1991, le gouvernement fédéral signifiait leur mise en disponibilité à plus de 500 employés des aéroports de Montréal à Mirabel et Dorval. Ce geste était immédiatement suivi d'une offre de réembauche d'ADM. À la suite de cette date importante, la signature d'une entente finale devait survenir dans les six mois. Au moment de la rédaction de cet article, on attend encore qu'Ottawa cesse de couper les cheveux en quatreet on conserve l'espoir' que l'entente définitive et finale soit signée avant Noël 1991 permettant ainsi une prise en charge effective des aéroports dans les tout premiers mois de 1992, après cinq longues années de démarches.

Que conclure de cette épopée? Il est extrềmement rare de voir un consensus politique régional perdurer pendant cinq ans avec une même ardeur et une volonté aussi persistante. Cela est dû à la conviction profonde de tous les partenaires qu' il fallait. à tout prix donner à Montréal le pouvoir décisionnel lui permettant de développer l'extraordinaire potentiel sconomique de ses aéroports sans que n'interviennent plus jamais l'exaspérante lenteur administrative d'Ottawaet les constantes brimades qui ont caractérisé son attitude depuis plus dequinze ans à l'égard des aéroports de Montréal. On peut enfin espérer contrôler le développement économique qu'on promettait pour nos aéroports il y a déjà vingt ans. $f$ 